\title{
Conversion of a diversity arrays technology marker differentiating wild and cultivated carrots to a co-dominant cleaved amplified polymorphic site marker*
}

\author{
Alicja Macko-Podgórni ${ }^{1 凶}$, Massimo lorizzo², Krzysztof Smółka1', Philipp W. Simon²,3 \\ and Dariusz Grzebelus ${ }^{1}$
}

\begin{abstract}
Cultivated carrot and its wild ancestor co-occur in most temperate regions of the world and can easily hybridize. The genetic basis of the process of domestication in carrot is not well understood. Recent results of an investigation on genetic diversity structure of cultivated and wild carrot and signatures for domestication using Diversity Arrays Technology (DArT) allowed identification of polymorphisms differentiating wild and cultivated accessions. We selected one of these polymorphisms, showing the strongest evidence for directional selection in the course of domestication, and converted it into a co-dominant cleaved amplified polymorphic site (CAPS) marker named cult. To achieve that, we designed site-specific primers anchored in sequences flanking the original DArT clone, amplified and sequenced the PCR products derived from cultivated and wild carrot. A Pstl restriction site present in the 'cultivated' variant and absent in the 'wild' was subsequently used for routine differentiation the two variants. We validated the cult marker on 88 accessions of cultivated and wild carrot, each represented by five individuals. The allelic variant associated with the wild phenotype was only rarely observed in cultivated carrot, mostly in purple-rooted accessions originating Turkey and Iran, possibly indicating that the physical association between the diagnostic polymorphism and the putative 'domestication gene' has been broken in a group of Eastern carrots.
\end{abstract}

Key words: carrot, domestication, DArT, CAPS

Received: 15 October, 2013; revised: 22 January, 2014; accepted: 21 February, 2014; available on-line: 17 March, 2014

\section{INTRODUCTION}

Cultivated carrot (Daucus carota subsp. sativus Hoffm.) is the most economically important species of the Apiaceae family. It is grown around the world in temperate and subtropical regions and constitutes the most significant source of $\beta$-carotenes, precursors of vitamin A. Wild carrot (D. carota subsp. carota L.) is widespread, presently appearing in temperate regions all over the world. Central Asia has been indicated as the place of origin of domesticated carrot. Cultivated and wild carrots can easily hybridize and a considerable amount of genetic variation is exhibited both among and within subspecies, with no apparent signature of domestication bottleneck (Iorizzo et al., 2013). Hybrids derived from cross-pollination of cultivated and wild carrot are characterized by woody, mostly white-colored root with low content of reducing sugars, resulting in bad taste and strong tendency for bolting (Wijnheijmer et al., 1989; Rong et al., 2013).

Several reports on the structure of genetic diversity in cultivated and wild carrot, using different strategies, were published (Shim \& Jorgensen 2000; Bradeen et al., 2002; Baranski et al., 2012; Iorizzo et al., 2013; Grzebelus et al., 2013). They allowed discrimination of three major groups comprising wild carrot and Western and Eastern types of cultivated carrot. In the latter study, a set of 900 non-redundant Diversity Array Technology (DAr'T) markers was used to identify signatures of domestication in the carrot genome. Twenty-seven markers were selected, among which marker crPt-895548 showed the strongest evidence for directional selection in the course of domestication (Grzebelus et al., 2013). As the marker allowed discrimination of cultivated and wild carrot, we decided to convert it into a reproducible, co-dominant, easy to handle, and cost-effective cleaved amplified polymorphic site (CAPS) marker. We tested performance of the converted marker on a wide collection of wild and cultivated carrot accessions of diverse origin.

\section{MATERIALS AND METHODS}

Plant materials. In total, 88 accessions representing 72 cultivated and 16 wild carrots of different origin were investigated. DNA was extracted from the fresh leaf tissue of using DNeasy Plant Mini Kit (Qiagen), as described by manufacturer. Each accession was represented by five individual plants.

PCR conditions and cloning of PCR products. PCR reactions were set up in $20 \mu \mathrm{l}$ comprising 20 ng genomic DNA, $0.5 \mu \mathrm{M}$ CULTex1i1F: 5'TGGATGGAAGAGAGGGAATG3' primer, $0.5 \mu \mathrm{M}$ CULTex1i1R: 5'TCCTGCCGAAGTATTCATCC3' primer, $0.25 \mathrm{mM}$ dNTPs, 1U DreamTaq DNA Polymerase (Thermo Scientific) and $1 \times$ DreamTaq Green Buffer. The following thermal conditions were applied: $94^{\circ} \mathrm{C}$

e-mail: mackoa@ogr.ar.krakow.pl

*Presented at the 5th Central European Congress of Life Sciences "EUROBIOTECH 2013", Kraków, Poland.

Abbreviations: DArT, diversity array technology; CAPS, cleaved amplified polymorphic site; ORF, open reading frame; SNP, single nucleotide polymorphism; AFLP, amplified fragment length polymorphisms; 1H NMR, proton nuclear magnetic resonance spectroscopy 
(2 min), 30 cycles of $94^{\circ} \mathrm{C}(30 \mathrm{~s}), 57^{\circ} \mathrm{C}(30 \mathrm{~s}), 68^{\circ} \mathrm{C}$ $(1 \mathrm{~min})$ and a final extension step of $68^{\circ} \mathrm{C}(5 \mathrm{~min})$. Products amplified from 3 wild carrots and 3 cultivated carrots were separated in 1\% agarose gels stained with ethidium bromide, purified with Wizard SV Gel and PCR Clean-Up (Promega), cloned into pGEM-T (Promega) and transferred into Escherichia coli strain DH10B. Plasmids extracted using Wizard SV Miniprep kit (Promega).

DNA sequencing. DArT clone crPt-895548 (Grzebelus et al., 2013) and cloned PCR products were Sanger-sequenced in a $5 \mu \mathrm{l}$ reaction including 1.75 $\mu \mathrm{l}$ of water, $1 \mu \mathrm{M}$ primer, $0.75 \mu \mathrm{l} 5 \times \mathrm{BigDye}^{\circledR} 3.1$ sequencing buffer, $0.5 \mu \mathrm{l}$ of $\mathrm{BigDye}{ }^{\circledR} 3.1$ ready reaction mix and $1 \mu$ l of PCR product, previously diluted 1:10 with water. Each insert was sequenced in both directions in separate reactions. Amplification conditions were: 25 cycles of $96^{\circ} \mathrm{C}$ for $10 \mathrm{~s}$, and $58^{\circ} \mathrm{C}$ for $2 \mathrm{~min}$, and a final step at $72^{\circ} \mathrm{C}$ for $5.0 \mathrm{~min}$. The sequences were analyzed on ABI 3730xl DNA Analyzer (Applied Biosystems). The sequences are available upon request.

Bioinformatic analysis. The sequence of DArT marker crPt-895548 was queried against the low coverage carrot genome assembly (Simon et al.,, unpublished) derived from data reported by Iorizzo et al. (2012) with blastn on a local BLAST server. The identified contig was searched for presence of coding regions using FGENESH (http://linux1.softberry.com/berry.phtml). The sequence was searched for conserved domains using Conserved Domain Database search at NCBI (MarchlerBauer et al., 2011).

Marker conversion and genotyping conditions. Primers were anchored in predicted exons flanking the intron in which crPt-895548 was localized, using Primer 3 (Rozen \& Skaletsky, 2000). Sequences derived from wild and cultivated carrots were manipulated and aligned in BioEdit (Hall 1999). PCR amplification for CAPS marker was set up as described above, followed by $1 \mathrm{~h}$ digestion in $37^{\circ} \mathrm{C}$ with $5 \mathrm{U}$ of Pst I (Thermo Scientific) and $1 \times \mathrm{O}$ buffer. The digested PCR products were separated in 1\% agarose gels, stained with ethidium bromide, and visualized under UV light.

\section{RESULTS}

305 bp-long sequence of crPt-895548 DArT marker differentiating wild and cultivated carrot showed a perfect match to 4770 bp-long genomic contig derived from B493 breeding line (Iorizzo et al., 2012) at positions 1163-1467. Prediction of putative coding sequences within the contig indicated presence of one complete ORF comprising five exons, located between 513 nt and 3698 nt (exon positions: 513-1020, 1571-1638, 20002131, 3169-3327, and 3465-3698) with a polyadenylation signal at 4097 nt (Fig. 1a). Sequence of crPt-895548 DArT marker was localized within the first intron of the predicted gene, thus we anchored primers in adjacent exons (primer positions: 514-533 and 1575-1594) and amplified that region from three wild and three cultivated carrot plants. Amplification products of expected size (ca. $1.1 \mathrm{~Kb}$ ) were observed for all individuals. Due to lack of distinguishable length differences, we sequenced all amplified products. We identified 16 SNPs and three indels (2, 10, and $16 \mathrm{bp})$. Six SNPs were located within the first exon, one within the second exon and nine within the intron. While SNP polymorphism in the exons was mostly observed among wild carrots, two SNPs and three indels located within the intron differentiated cultivated and wild carrots. The sequence of the PstI restriction site located within an indel (starting from 1463 nt) and present only in the cultivated variant (Fig. 1b) caused the previously reported DArT polymorphism (Grzebelus et al., 2013). Here, we used it for discrimination between Pst I-digested PCR products representing cultivated and wild variants (Fig. 2). That way we converted DAr'T marker into a co-dominant CAPS marker we named cult (for cultivated).

We validated the cult marker with 88 accessions of cultivated and wild carrot, each represented by five individuals. In case of cultivated carrots, 58 of 72 accessions carried cult variant expected for cultivated carrot (homozygous for the 'cultivated' variant). However, in 14 cultivated accessions the 'wild' variant was observed in at least one plant (Fig. 3). Notably, in case of 7262B inbred line all five plants carried the homozygous 'wild' cult variant. On the other hand, we observed presence of the 'cultivated' allelic variant in five of 16 wild car-

a

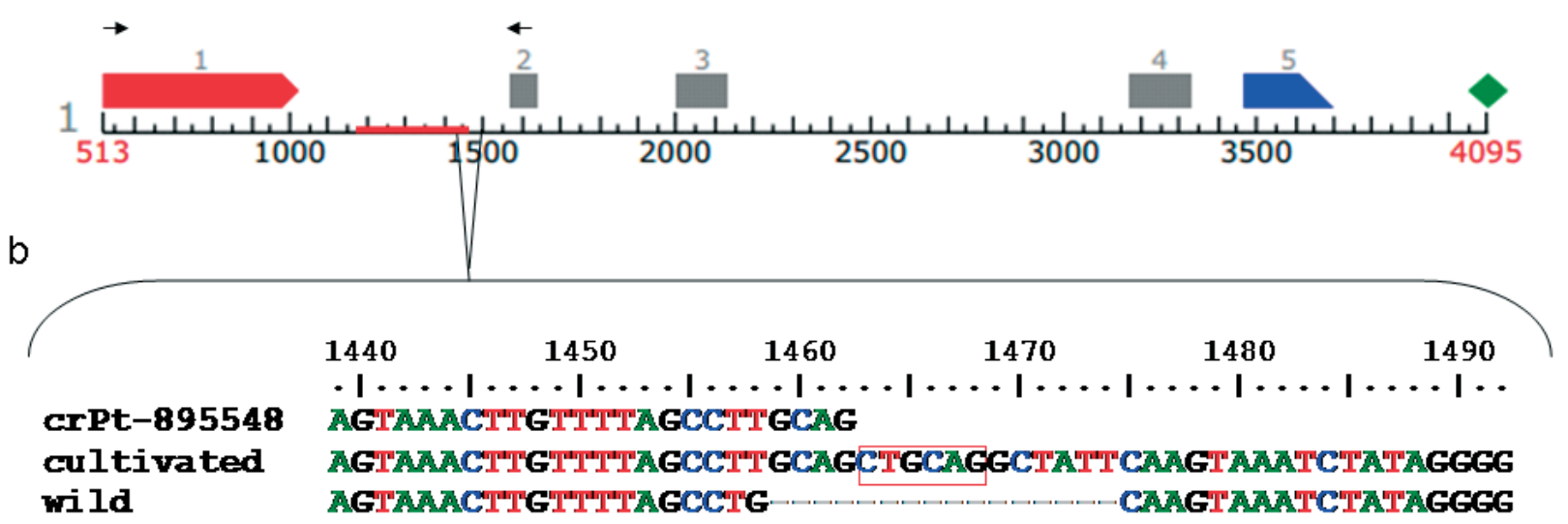

Figure 1. Scheme of the predicted open reading frame (a) and the indel in the 5' region of crPt-895548 DArT clone with Pstl restriction site present only in the variant typical for cultivated carrot (b).

Red arrow represents the first exon; gray boxes represent internal exons; blue box represents the last exon; green diamond represents polyA site; the region of crPt-895548 DArT marker is highlighted in red, arrows indicate primer anchor sites; red rectangle indicates Pst restriction site. 


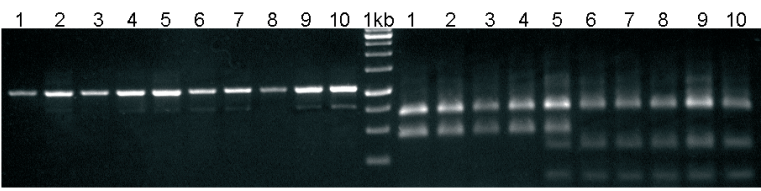

Figure 2. Products of PCR amplification of the $5^{\prime}$ region flanking crPt-895548 DArT clone before (left panel) and after digestion with Pstl (right panel).

Lanes: $1-D$. carota subsp. carota (Poland), $2-D$. carota subsp. carota (Russia), $3-D$. carota subsp. carota (Slovakia), $4-D$. carota subsp. azoricus (Portugal), $5-D$. carota subsp. gummifer (Great Britain), 6 - Persia No 242 (Iran), 7 - Pusa Kesar (India), 8 - Tropical (Brazil), 9 - Long Red (Ethiopia), 10 - 0493B (USA).

rot accessions. Within D. carota subsp. gummifer and one population of $D$. carota subsp. carota from Chile two and four plants respectively were heterozygous, while three accessions - D. carota subsp. maximus and two populations of $D$. carota subsp. carota from Israel and Russia comprised individuals both heterozygous or homozygous for the 'cultivated' variant (Fig. 2).

\section{DISCUSSION}

A range of molecular marker systems was used to study carrot diversity. Dominant AFLP (Shim and Jorgensen, 2000) and co-dominant SNP (Iorizzo et al., 2013) markers allowed characterization of genetic diversity structure and discriminated wild and cultivated accessions but no markers linked to the cultivated/ wild type were reported. Only recently, a large scale genotyping of 159 accessions including 65 wild carrot populations with 900 non-redundant DArT dominant markers allowed identification of several markers showing possible signatures for domestication. Among them, DArT marker crPt-895548 was identified as being the most likely located in a genomic region involved in domestication (Grzebelus et al., 2013). It was localized within intron 1 of a predicted gene what may suggest a relationship between of the putative gene product func- tion and domestication. However, explanation of that issue requires additional investigation.

The possibility to distinguish wild and cultivated carrot is of high importance especially for improvement of modern carrot breeding. Although carrot domestication took place more than 1000 years ago, gene flow from wild to cultivated carrot has been still observed worldwide and may not only contaminate breeding materials but also cause problems in the context of a gene flow following possible future introduction of genetically modified carrot cultivars. On the other hand, easy hybridization of cultivated and wild carrot may facilitate introgression of new traits, such as cytoplasmic male sterility or resistance to serious pests and significant diseases, into the cultivated gene pool (Grzebelus et al., 2011).

Visual selection of outcrosses based on the morphological markers does not allow reliable identification of hybrids (Grebenstein et al., 2013). Root color-based selection seems to be the most effective for screening breeding materials but it is time-consuming and useful only for orange-rooted cultivars, as hybrids of carrots with yellow and purple root color only partially lose their pigmentation as a result of cross-pollination with wild carrot (Simon, 1996). Analysis of metabolite content with $1 \mathrm{H}$ NMR allowed clustering cultivated and wild carrot, but all hybrids were grouped with wild parental plants (Grebenstein et al.,, 2011). For these reasons, cost-effective and time-efficient method of identification of hybrid plants would be very valuable. Here, we reported on a molecular marker that could be easily employed in routine screening of wild and cultivated carrot population for their hybrid status.

The incidence of the 'cultivated' variant of the cult polymorphism in wild carrot accessions reported here was higher than that observed by Grzebelus et al. (2013) with the corresponding DAr'T marker. Possibly, some wild accessions were in fact wild/cultivated hybrids, as they showed an intermediate phenotype resulting in a slightly swollen root (not shown). A broader sampling of wild carrot is necessary to sys-

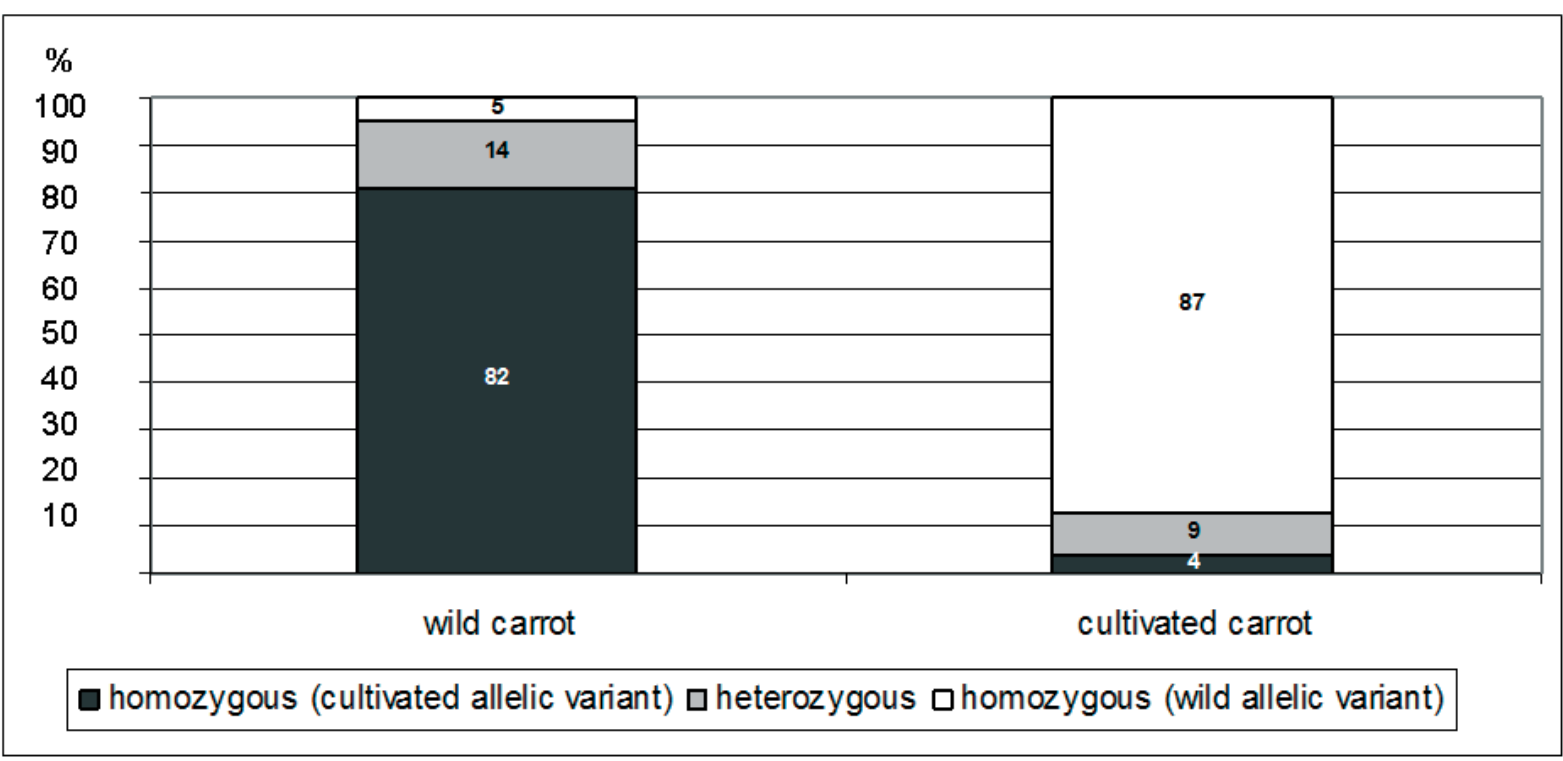

Figure 3. Distribution of 'cultivated' and 'wild' allelic variants of the cult marker in 72 and 16 accessions of cultivated and wild carrot accessions, respectively, each accession was represented by five individuals. 
tematically verify the frequency of the 'cultivated' variant in the wild gene pool.

In few cases we observed presence of 'wild' cult variant within plants of cultivated accessions. Most of them were landraces and open-pollinated cultivars from Asia (mostly Turkey) with characteristics typical for Eastern cultivated carrot, e.g. purple roots. It is likely that they were poorly monitored for pollination occurring with contribution of wild relatives. However, presence of the homozygous 'wild' cult variant in all five plants of $7262 \mathrm{~B}$ inbred line requires additional attention. The purple-rooted $7262 \mathrm{~B}$ inbred line originated from a cross between a Turkish accession highly variable for root color and shape and a dark orange-rooted breeding line. It is characterized by very dark purple outer phloem and orange-colored core, vigorous, medium green tops, with strong attachment to the root and some tendency for early flowering (Simon et al., 1997), apparently resembling Eastern type carrot. Thus, it might be possible that the physical linkage between the cult polymorphism and the 'domestication gene' has been broken in a group of purple-rooted Eastern carrots.

In general, results reported here and those presented previously by Grzebelus et al., (2013) suggest that the 'cultivated' variant of the cult locus, rare in wild gene pool, was enriched in the cultivated gene pool in the course of domestication.

\section{Acknowledgements}

The research was funded by the Polish National Science Center, project no. 2012/05/B/NZ9/03401 awarded to DG.

\section{REFERENCES}

Baranski R, Maksylewicz-Kaul A, Nothnagel T, Cavagnaro PF, Simon PW, Grzebelus D (2012) Genetic diversity of carrot (Daucus carota L.) cultivars revealed by analysis of SSR loci. Genet Resour Crop Evol 59: $163-170$

Bradeen JM, Bach IC, Briard M, LeClerc V, Grzebelus D, Senalik DA, Simon PW (2002) Molecular diversity analysis of cultivated carrot (Daucus carota L.) and wild Daucus populations reveals a genetically nonstructured composition. I Amer Soc Hort Sci 127: 383-391.

Grebenstein C, Choi YH, Rong J, Jong TJ, Tamis WLM (2011) Metabolic fingerprinting reveals differences between shoots of wild and cultivated carrot (Daucus carota L.) and suggests inheritance or wild trait dominance in hybrids. Phytochemistry 72: 1341-1347.

Grebenstein C, Kos SP, Jong TJ, Tamis WL, Snoo GR (2013) Morphological markers for the detection of introgression from cultivated into wild carrot (Daucus carota L.) reveal dominant domestication traits. Plant Biol 15: 531-540.

Grzebelus D, Baranski R, Spalik K, Allender C, Simon PW (2011) Daucus. In Wild Crop Relatives: Genomic and Breeding Resources. Kole C, eds, pp 91-113. Springer Berlin Heidelberg.

Grzebelus D, Iorizzo M, Senalik D, Ellison S, Cavagnaro P, MackoPodgorni A, Heller-Uszynska K, Kilian A, Nothnagel T, Simon PW, Baranski R (2014) Diversity, genetic mapping, and signatures of domestication in the carrot (Daucus carota L.) genome, as revealed by Diversity Arrays Technology (DAr'T) markers. Mol Breed. 33: $625-637$.

Hall TA (1999) BioEdit: a user-friendly biological sequence alignment editor and analysis program for Windows 95/98/NT. Nucl Acids Symp 41: 95-98.

Iorizzo M, Senalik D, Szklarczyk M, Grzebelus D, Spooner D, Simon PW (2012) De novo assembly of the carrot mitochondrial genome using next generation sequencing of whole genomic DNA provides first evidence of DNA transfer into an angiosperm plastid genome. BMC Plant Biol 12: 61.

Iorizzo M, Senalik DA, Ellison SL., Grzebelus D, Cavagnaro PF, Allender C, Brunet J, Spooner DM, Van Deynze A, Simon PW (2013) Genetic structure and domestication of carrot (Daucus carota subsp. sativus)(Apiaceae). Am J Bot 100: 930-938.

Marchler-Bauer A, Lu S, Anderson JB, Chitsaz F, Derbyshire MK, DeWeese-Scott C, Fong JH, Geer LY, Geer RC, Gonzales NR, Gwadz M, Hurwitz DI, Jackson JD, Ke Z, Lanczycki CJ, Lu F, Marchler GH, Mullokandov M, Omelchenko MV, Robertson CL, Song JS, Thanki N, Yamashita RA, Zhang D, Zhang N, Zheng C, Bryant SH (2010) CDD: a Conserved Domain Database for the functional annotation of proteins. Nucl Acids Res 39: D225-D229.

Rong J, Xu S, Meirmans PG, Vrieling K (2013) Dissimilarity of contemporary and historical gene flow in a wild carrot (Daucus carota) metapopulation under contrasting levels of human disturbance: implications for risk assessment and management of transgene introgression. Ann Bot 112: 1361-1370.

Rozen S, Skaletsky HJ (2000) Primer3 on the WWW for general users and for biologist programmers. In Bioinformatics methods and protocols: methods in molecular biology. Krawetz S, Misener S, eds, pp 365-386. Humana Press, Totowa, NJ, Source code available at http://fokker. wi.mit.edu/primer3/

Shim SI, Jorgensen RB (2000) Genetic structure in cultivated and wild carrots (Daucus carota L.) revealed by AFLP analysis. Theor Appl Genet 101: 227-233.

Simon PW (1996) Inheritance and expression of purple and yellow storage root color in carrot. J Hered 87: 63-66.

Simon PW, Rubatzky VE, Bassett MJ, Strandberg OJ, White JM (1997) B7262, purple carrot inbred. Hort Science 32: 146-147.

Wijnheijmer EHM, Brandenburg WA, Ter Borg SJ (1989) Interaction between wild and cultivated carrots (Daucus carota L.) in the Netherlands. Euphytica 40: 147-154. 